\title{
A novel way to probe distribution amplitudes of neutral mesons in $e^{+} e^{-}$annihilation.
}

\author{
N.A. Kivel ${ }^{1,2}$, M. V. Polyakov ${ }^{1,2}$ \\ ${ }^{1}$ Institute for Theoretical Physics II, Ruhr-University, 44780 Bochum, Germany \\ ${ }^{2}$ Petersburg Nuclear Physics Institute, 188300 Gatchina, Russia
}

\begin{abstract}
We derive the amplitude for the process $e^{+} e^{-} \rightarrow \pi^{0} \pi^{0}$ at large invariant energy. The process goes through the two-photon exchange and its amplitude is expressed in terms of the convolution integral which depends on the shape of the pion distribution amplitude (DA) and the centre of mass scattering angle. Remarkable feature of the integral is that it is very sensitive to the end-point behaviour of the pion DA it starts to diverge if pion DA nullifies at the end-point as $\sqrt{x}$ or slower. That makes the $e^{+} e^{-} \rightarrow \pi^{0} \pi^{0}$ process unique probe of the shape of the meson DAs. The estimated cross section is rather small, for $\sqrt{s}=3 \mathrm{GeV}$ it ranges from a fraction of femtobarn (for the asymptotic DA) to couple of femtobarn (for the ChernyakZhitnitsky DA). The observation of the process $e^{+} e^{-} \rightarrow \pi^{0} \pi^{0}$ with the cross section higher as estimated here would imply very unusual form of the pion DA, e.g. the flat one. The derived amplitude can be easily generalized to other processes like $e^{+} e^{-} \rightarrow \sigma \sigma, K_{S} K_{S}, \eta \eta, \eta^{\prime} \eta, \pi^{0} f_{2}$, etc.
\end{abstract}

1. Distribution amplitudes (DAs) of mesons are the basic non-perturbative objects which describe longitudinal momentum distributions in the lowest $q \bar{q}$ Fock component of the meson light-cone wave function. The meson DA is defined as the matrix element of the QCD operator on the light-cone sandwiched between vacuum and the meson. For instance, the pion* $\mathrm{DA} \phi(z)$ is defined as the following matrix element:

$$
\left\langle 0\left|\bar{d}(n) \gamma_{\mu} n^{\mu} \gamma_{5}[n,-n] u(-n)\right| \pi^{+}(P)\right\rangle=i \sqrt{2} f_{\pi}(n \cdot P) \int_{0}^{1} d x e^{i(2 x-1) P \cdot n} \phi(x)
$$

Here $f_{\pi}=92.4 \mathrm{MeV}$ is the pion decay constant, $n^{\mu}$ is a light-like 4 -vector $\left(n^{2}=0\right)$, and

$$
[n,-n] \equiv \mathrm{P} \exp \left[\int_{-1}^{1} d t n^{\mu} A_{\mu}(t n)\right]
$$

denotes the path-ordered exponential, required by gauge invariance. The pion DA is normalized by $\int_{0}^{1} d x \phi(x)=1$ and it possesses the symmetry $\phi(1-x)=\phi(x)$.

The DA is the universal object which enters description of many hard exclusive processes, such as $\gamma^{*} \gamma \rightarrow \pi^{0}, \gamma \gamma \rightarrow 2 \pi$, pion form-factor, etc [1. One of classical examples is the pion form factor which can be measured in $e^{+} e^{-} \rightarrow \pi^{+} \pi^{-}$processes. In the leading order in $\alpha_{\mathrm{em}}$ the process occurs through the one photon exchange. The asymptotic of the amplitude at large invariant energy $\left(\sqrt{s} \gg \Lambda_{\mathrm{QCD}}\right)$ of colliding leptons can be written, up to the corrections of the order $\alpha_{s}^{2}$, as:

*In this paper we shall discuss and present all calculations for the case of the pions, the generalization for other mesons is trivial. 


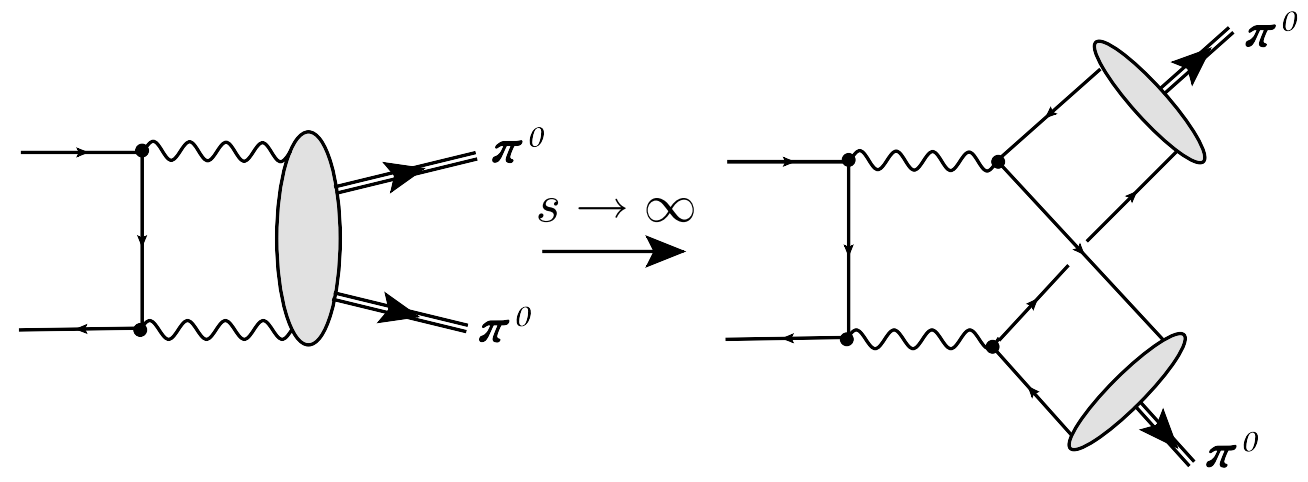

Figure 1: Two photon exchange diagram for the $e^{+} e^{-} \rightarrow \pi^{0} \pi^{0}$ process. On the right part of the figure the leading QCD diagram is shown. There is another leading diagram with crossed photons which we do not show here.

$A\left(e^{+}(k) e^{-}\left(k^{\prime}\right) \rightarrow \pi^{+}(p) \pi^{-}\left(p^{\prime}\right)\right)=\frac{\alpha_{\mathrm{em}}\left(e_{u}-e_{d}\right)}{9} \frac{4 \pi \alpha_{s} f_{\pi}^{2}}{s^{2}}\left|\int_{0}^{1} d x \frac{\phi(x)}{x(1-x)}\right|^{2} \bar{l}\left(k^{\prime}\right) \not P l(k)$.

Here $e_{u}, e_{d}$ are quark charges of the corresponding flavour in the units of the positron charge and we introduced the relative pion momentum $P^{\mu}=\left(p-p^{\prime}\right)^{\mu}$. The strong coupling constant $\alpha_{s}$ appears due to the hard gluon exchange needed to make quarks and antiquarks collinear to the final pions.

2. Now if we turn to the process $e^{+} e^{-} \rightarrow \pi^{0} \pi^{0}$ the one photon exchange does not contribute to the amplitude as the final state has positive $\mathrm{C}$-parity. The leading contribution is due to the two-photon exchange, see Fig. 1. At large collision energy the $\pi^{0} \mathrm{~s}$ are produced favourably by two $q \bar{q}$ pairs flying along two different light-cone directions with quark and antiquark having small relative transverse momentum, $k_{\perp}^{2} / s \ll 1$. The simplest diagram to create such pairs is shown on the right side of Fig. $1 \oplus$.

An analysis of the diagram in Fig. 1 gives that the dominant virtuality of the intermediate photons is of the order $\sim s$. That indicates that the $q \bar{q}$ pairs are produced at a short distance and possible QCD corrections are suppressed by small $\alpha_{s}$ at the scale of the order of invariant energy $\sim \sqrt{s}$.

Calculating the corresponding diagrams (see Fig. 1) we obtain the following result for the amplitude:

$$
\lim _{s \rightarrow \infty} A\left(e^{+}(k) e^{-}\left(k^{\prime}\right) \rightarrow \pi^{0}(p) \pi^{0}\left(p^{\prime}\right)\right)=\frac{\alpha_{\mathrm{em}}^{2}\left(e_{u}^{2}+e_{d}^{2}\right)}{3} \frac{8 \pi^{2} f_{\pi}^{2}}{s^{2}} G\left(\cos \theta_{\mathrm{cm}}\right) \bar{l}\left(k^{\prime}\right) P p l(k),
$$

where the dependence on the centre of mass scattering angle $\theta_{\mathrm{cm}}$ is given by the function $G\left(\cos \theta_{\mathrm{cm}}\right)$. This function is given by the following convolution integral of the pion DA:

\footnotetext{
${ }^{\dagger}$ Note that for production of neutral vector mesons there is another type of diagrams in which two photons are transformed into the vector meson independently of each other. Such case has been considered recently in Ref. [2] and we do not dwell on it here.
} 


$$
G(z)=z \int_{0}^{1} d x \int_{0}^{1} d y \frac{\phi(x)}{x(1-x)} \frac{\phi(y)}{y(1-y)} \frac{[x(1-x)+y(1-y)]}{\left[(x+y-2 x y)^{2}-z^{2}(x-y)^{2}\right]} .
$$

This function is obviously odd in $\cos \theta_{\mathrm{cm}}$, as it should be on the basis of Bose symmetry. Also we note that Eq. (4) is derived under the assumption that $s \sin ^{2} \theta_{\mathrm{cm}} \gg \Lambda_{\mathrm{QCD}}^{2}$, that corresponds to the kinematical range of the Mandelstam variables $|t| \sim|u| \sim s \gg \Lambda_{\mathrm{QCD}}^{2}$. It is easy to see that the convolution integral (5) is divergent if $\sin \theta_{\mathrm{cm}}$ goes to zero.

Comparing the annihilation amplitude into $\pi^{+} \pi^{-}$(3) with that for $\pi^{0} \pi^{0}$ (44), we see that the latter has suppression by $\alpha_{\mathrm{em}} \approx 1 / 137$, however it has the same scaling behaviour in $1 / s$. The annihilation into neutral pions has no suppression by $\alpha_{s}$. The factor $\alpha_{s}$ in Eq. (3) is due to the hard gluon exchange needed to make relative transverse momentum in each $q \bar{q}$ pairs producing pions small, in the case of annihilation into $\pi^{0} \pi^{0}$ one does not need gluon exchange for that. The expression (44) with the replacement $\left(e_{u}^{2}+e_{d}^{2}\right) \rightarrow 8 e_{u} e_{d}$ provides an $\alpha_{\mathrm{em}}$ correction for the process $e^{+} e^{-} \rightarrow \pi^{+} \pi^{-}$and can be used for more precise extraction of the pion e.m. form factor from the annihilation process. Similar to considered here, a mechanism for the $\alpha_{\mathrm{em}}$ corrections in the elastic lepton scattering on the nucleon has been considered recently in Refs. [3, 4].

A remarkable property of the convolution integral (5) is that it is sensitive to the end point behaviour of the pion DA. It is divergent if the pion DA $\phi(x)$ at small $x$ behaves as $\phi(x) \sim x^{\alpha}$ with $\alpha \leq \frac{1}{2}$. Similar feature is possessed by the convolution integrals which enter QCD description of the exclusive charmonia decays [5]. All other known hard exclusive processes contain convolution integrals which diverge only if the pion DA is not zero at the end points, i.e. if $\alpha \leq 0$. Recent measurements of the $\gamma^{*} \gamma \rightarrow \pi^{0}$ by the BaBar collaboration [6] show surprising rise of the scaled $\gamma \pi$ form factor at large $Q^{2}$. In Refs. [7, 8] this phenomenon is explained by the flat pion DA. The measurement of the $e^{+} e^{-}$annihilation into two $\pi^{0} \mathrm{~s}$ will allow to check this hypothesis due to the sensitivity of the convolution integral (5) to the end-point behaviour of the pion DA. One would expect the change of the scaling behaviour in $1 / s$ for the cross-section - slower than $1 / s^{3}$.

3. The cross section of the process $e^{+} e^{-} \rightarrow \pi^{0} \pi^{0}$ computed from the amplitude (44) has the form:

$$
\frac{d \sigma}{d \cos \theta_{\mathrm{cm}}}=\frac{25}{1458} \frac{\alpha_{\mathrm{em}}^{4} \pi^{3} f_{\pi}^{4}}{s^{3}} \sin ^{2} \theta_{\mathrm{cm}}\left|G\left(\cos \theta_{\mathrm{cm}}\right)\right|^{2} .
$$

Here the function $G\left(\cos \theta_{\mathrm{cm}}\right)$ is given by the convolution integral (51) and depends on the form of the pion DA. In order to show the sensitivity of the cross section (66) to the pion DA, we plotted in Fig. 2 the cross section as the function of the scattering angle at the invariant energy $\sqrt{s}=3 \mathrm{GeV}$ for three choices of the pion DA: the non-relativistic one $\phi(x)=\delta\left(x-\frac{1}{2}\right)$, the asymptotic one $\phi(x)=6 x(1-x)$ and for the pion DA a'la Chernyak-Zhitnitsky (CZ) $\phi(x)=30 x(1-x)(2 x-1)^{2}$ [5]. As it is expected the crosssection depends very strongly on the form of the pion DA, however the cross section is very small- it ranges from a fraction of femtobarn for the asymptotic DA to a couple of femtobarns for the CZ DA. It is challenge to measure such small cross-section, also an observation of the $e^{+} e^{-} \rightarrow \pi^{0} \pi^{0}$ process with considerably higher cross section (say, at the level of 10 th of femtobarn at $\sqrt{s}=3 \mathrm{GeV}$ ) would signal about very unusual pion 


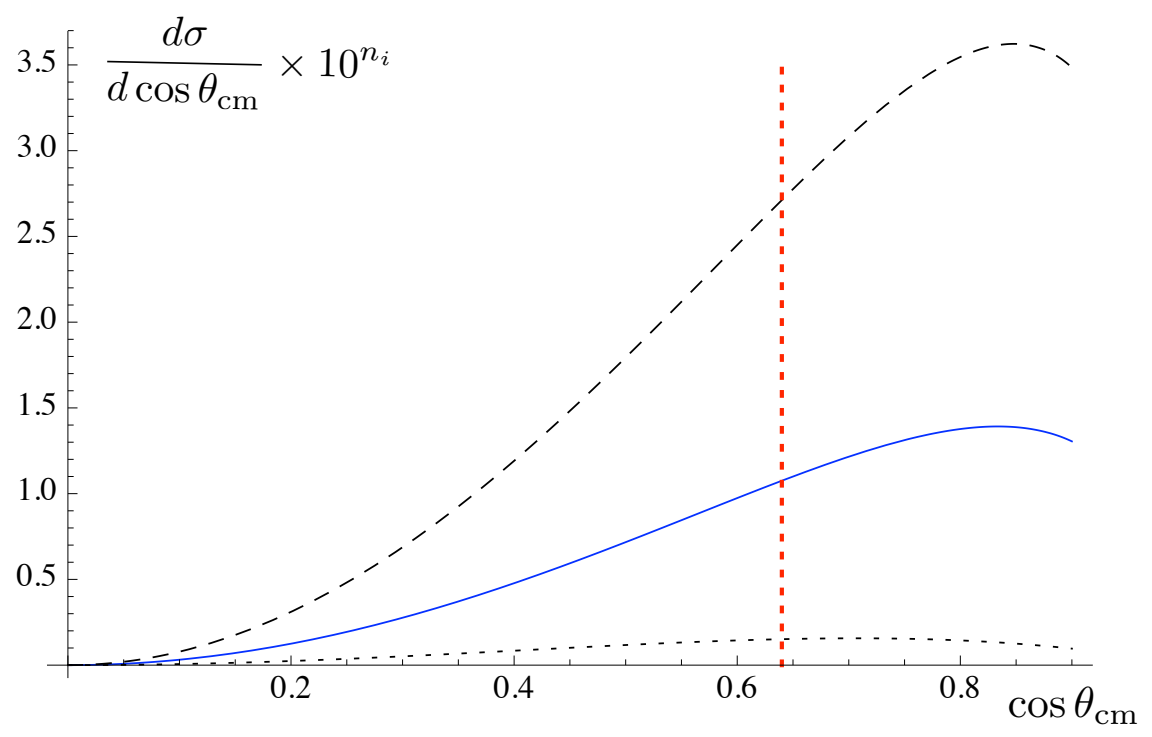

Figure 2: The angular dependence of the cross section in femtobarns of the $e^{+} e^{-} \rightarrow \pi^{0} \pi^{0}$ process at $\sqrt{s}=3 \mathrm{GeV}$. The dashed line corresponds to the model for pion DA a'la Chernyak-Zhitnitsky $\phi(x)=30 x(1-x)(2 x-1)^{2}$ (scale factor exponent $n_{C Z}=0$ ), the solid line corresponds to the asymptotic pion DA $\phi(x)=6 x(1-x)$ (scale factor exponent $n_{\text {asy }}=1$ ), and the dashed line with short dashes corresponds to the cross section with nonrelativistic pion DA $\phi(x)=\delta\left(x-\frac{1}{2}\right)$ (scale factor exponent $n_{N R}=1$ ). By the vertical line we show the range of angles at which the whole formalism can be applied, it corresponds to the range of kinematical variable $|t|,|u| \geq 1.5 \mathrm{GeV}^{2}$.

DA, e.g. the flat one. In Fig 2 by the vertical line we show the range of angles at which the whole formalism can be applied, it corresponds to the range of kinematical variable $|t|,|u| \geq 1.5 \mathrm{GeV}^{2}$ at $\sqrt{s}=3 \mathrm{GeV}$.

Let us now to study the shape of the angular dependence of the cross section. For that we present the cross section (6) in the following form:

$$
\frac{d \sigma}{d \cos \theta_{\mathrm{cm}}}=\frac{25}{1458} \frac{\alpha_{\mathrm{em}}^{4} \pi^{3} f_{\pi}^{4}}{s^{3}} N S\left(\theta_{\mathrm{cm}}\right)
$$

where the shape function $S\left(\theta_{\mathrm{cm}}\right)$ and normalization constant $N$ are given in terms of the convolution integral (5) as follows:

$$
S\left(\theta_{\mathrm{cm}}\right)=\frac{1}{N} \sin ^{2} \theta_{\mathrm{cm}}\left|G\left(\cos \theta_{\mathrm{cm}}\right)\right|^{2}, \quad N=\int_{-1}^{1} d\left(\cos \theta_{\mathrm{cm}}\right) \sin ^{2} \theta_{\mathrm{cm}}\left|G\left(\cos \theta_{\mathrm{cm}}\right)\right|^{2} .
$$

The total cross section is obviously expressed in terms of the normalization constant $N$ as follows:

$$
\sigma_{\mathrm{tot}}=\frac{25}{1458} \frac{\alpha_{\mathrm{em}}^{4} \pi^{3} f_{\pi}^{4}}{s^{3}} N
$$


In order to study the dependence of the normalization and the shape function on the form of the pion DA, let us choose simple one parametric Ansatz for the pion DA taking into account only the first term in the Gegenbauer expansion:

$$
\phi(x)=6 x(1-x)\left(1+a_{2} C_{2}^{\frac{3}{2}}(2 x-1)\right),
$$

where parameter $a_{2}$ we treat as a free. The result for the convolution integral (5) with the Ansatz (10) is the following:

$$
\begin{aligned}
\frac{G(z)}{36} & =\ln \left(\frac{1+z}{1-z}\right)+a_{2} g_{1}(z)+a_{2}^{2} g_{2}(z) \\
g_{1}(z) & =\left(15 z^{2}-8\right) \ln \left(\frac{1+z}{1-z}\right)+\frac{15}{4} z\left(\pi^{2}\left(1-z^{2}\right)+\left(1-z^{2}\right) \ln ^{2}\left(\frac{1+z}{1-z}\right)-4\right) \\
g_{2}(z) & =-\frac{3}{4}\left(5 z\left(60 z^{2}+3 \pi^{2}\left(5 z^{4}-8 z^{2}+3\right)-56\right)+15 z\left(5 z^{4}-8 z^{2}+3\right) \ln ^{2}\left(\frac{1+z}{1-z}\right)\right. \\
& \left.-4\left(75 z^{4}-95 z^{2}+22\right) \ln \left(\frac{1+z}{1-z}\right)\right)
\end{aligned}
$$

The result for the normalization constant $N$ in Eq. (8) is the following:

$$
\begin{aligned}
\frac{N}{(36)^{2}} & =\frac{4}{9}\left(\pi^{2}-6\right)+\frac{8}{9}\left(\pi^{2}+3\right) a_{2}+\frac{2}{63}\left(96 \pi^{4}-800 \pi^{2}-399\right) a_{2}^{2} \\
& -\frac{4}{77}\left(576 \pi^{4}-6644 \pi^{2}+8547\right) a_{2}^{3}+\left(\frac{94464}{1001} \pi^{4}-\frac{7792}{7} \pi^{2}+1824\right) a_{2}^{4} \\
& \approx 1.72\left(1+6.65 a_{2}+19.50 a_{2}^{2}+27.76 a_{2}^{3}+17.55 a_{2}^{4}\right) .
\end{aligned}
$$

One sees that the normalization of the cross section has strong dependence on the parameter $a_{2}$. This dependence is stronger than the corresponding dependence of the cross section for $e^{+} e^{-} \rightarrow \pi^{+} \pi^{-}$reaction.

The shape function $S\left(\theta_{\mathrm{cm}}\right)$ is shown in Fig. 3, where we plot this function for several values of the parameter $a_{2}$. One sees that for positive values of $a_{2}$ the shape function is almost indistinguishable from that for the asymptotic pion DA. One can check that for the positive values of $a_{2}$ the corresponding shape functions can be hardly distinguished from each other. For the negative values of $a_{2}$ the shape function have different behaviour, see the solid line in Fig. 3. That means that the measurements of the shape function would allows us to find out if the pion DA is wider or narrower than the asymptotic one. The knowledge of the shape function does not allow to determine unambiguously pion DA. One can easily give examples of very different pion DAs that leads to the same shape functions.

4. In summary, we derived the amplitude for the process $e^{+} e^{-} \rightarrow \pi^{0} \pi^{0}$ at large invariant energy. The main result is presented by Eq. (44). The process goes through the twophoton exchange and its amplitude is expressed in terms of the convolution integral (5) that depends on the shape of the pion DA and cm scattering angle. Remarkable feature of that integral is that it is very sensitive to the end-point behaviour of the pion DA it starts to diverge if pion DA nullifies at the end-point as $\sqrt{x}$ or slower. The estimated cross section is rather small, that is a challenge for the experiments. The observation of 


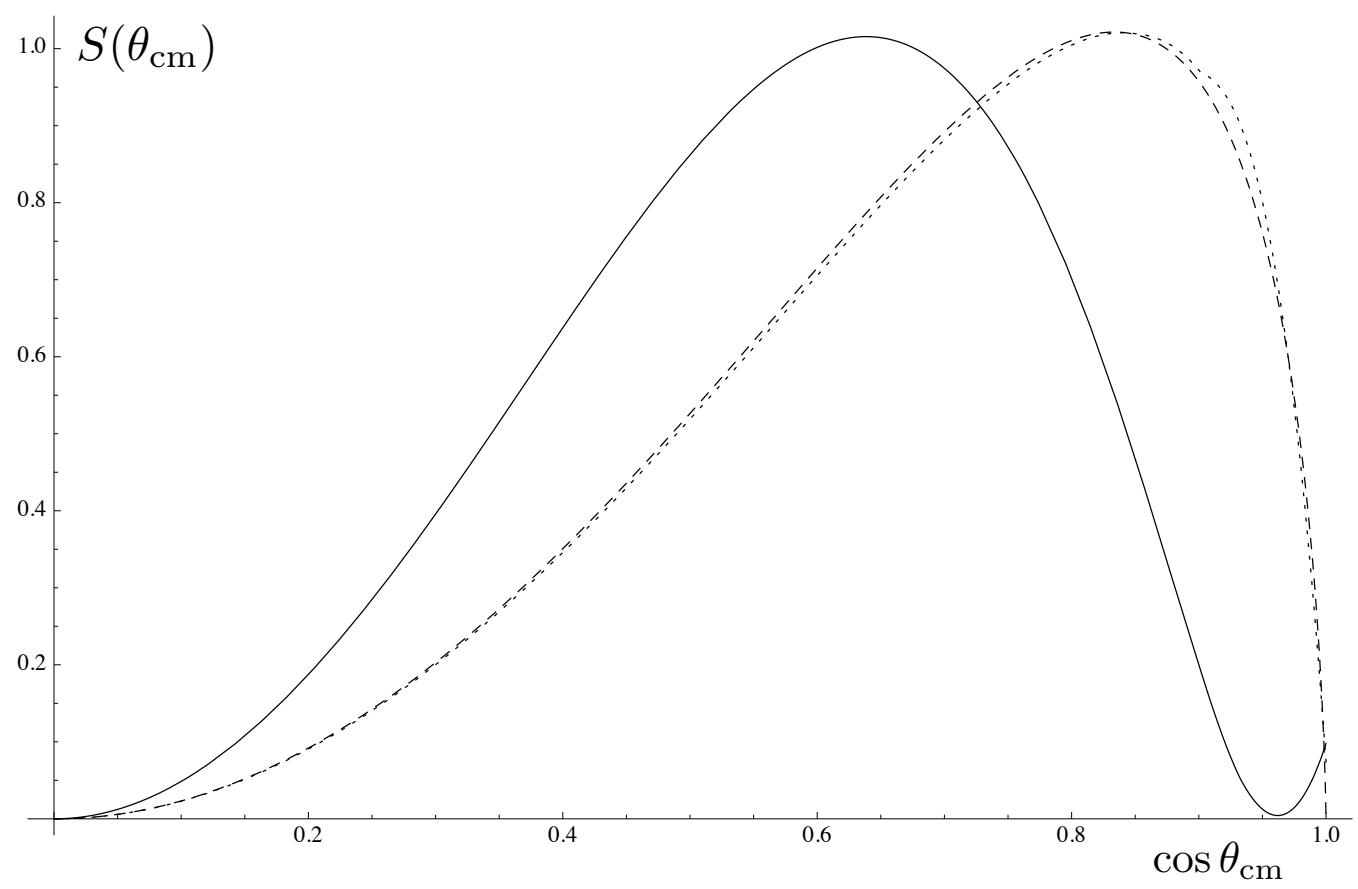

Figure 3: The shape function (8) as a function of the scattering angle. The dotted and dashed lines (almost indistinguishable ) correspond to the values of the parameter $a_{2}$ in Eq. (10) $a_{2}=0$ and $a_{2}=1$. The solid line corresponds to $a_{2}=-1 / 2$.

the process $e^{+} e^{-} \rightarrow \pi^{0} \pi^{0}$ with the cross section higher as estimated here would imply very unusual form of the pion DA, e.g. the flat one. The derived amplitude can be easily generalized to other processes like $e^{+} e^{-} \rightarrow \sigma \sigma, K_{S} K_{S}, \eta \eta, \eta^{\prime} \eta, \pi^{0} f_{2}$, etc.

\section{Acknowledgements}

Discussions with D. Mueller, M. Strikman, M. Vanderhaeghen and A. Vladimirov are greatly appreciated. This work was supported in parts by BMBF and by the Deutsche Forschungsgemeinschaft.

\section{References}

[1] V. L. Chernyak, A. R. Zhitnitsky and V. G. Serbo, JETP Lett. 26 (1977) 594 [Pisma Zh. Eksp. Teor. Fiz. 26 (1977) 760];

A. V. Radyushkin, JINR-P2-10717 (1977); English translation: arXiv:hep-ph/0410276.

G. P. Lepage and S. J. Brodsky, Phys. Lett. B 87 (1979) 359;

G. P. Lepage and S. J. Brodsky, Phys. Rev. D 22, 2157 (1980);

A. V. Efremov and A. V. Radyushkin, Theor. Math. Phys. 42, 97 (1980) [Teor. 
Mat. Fiz. 42, 147 (1980)];

A. V. Efremov and A. V. Radyushkin, Phys. Lett. B 94, 245 (1980).

[2] M. Davier, M. E. Peskin and A. Snyder, arXiv:hep-ph/0606155.

[3] D. Borisyuk and A. Kobushkin, Phys. Rev. D 79 (2009) 034001 arXiv:0811.0266 [hep-ph]].

[4] N. Kivel and M. Vanderhaeghen, arXiv:0905.0282 [hep-ph].

[5] V. L. Chernyak and A. R. Zhitnitsky, Nucl. Phys. B 201 (1982) 492 [Erratum-ibid. B 214 (1983) 547].

[6] B. Aubert [The BABAR Collaboration], arXiv:0905.4778 [hep-ex].

[7] A. V. Radyushkin, arXiv:0906.0323 [hep-ph].

[8] M. V. Polyakov, arXiv:0906.0538 [hep-ph]. 